5th International Workshop on Astronomy and

Relativistic Astrophysics (IWARA2011)

International Journal of Modern Physics: Conference Series

Vol. 18 (2012) 63-66

(C) World Scientific Publishing Company

DOI: $10.1142 / \mathrm{S} 2010194512008215$

\title{
HIGH RESOLUTION SPECTROPOLARIMETRY OF THE SOLAR TWINS
}

\author{
THARCISYO DUARTE and J. D. DO NASCIMENTO JR. \\ Departamento de Física Teórica e Experimental, \\ Universidade Federal do Rio Grande do Norte \\ Natal, R.N. 59072-970, Brazil \\ tssduarte@gmail.com; \\ dias@dfte.ufrn.br
}

\begin{abstract}
The study of solar twins offers a unique opportunity to investigating the solar magnetic field over longer timescales. Supported by the new generation of stellar spectropolarimeters (ESPaDOnS@CFHT, NARVAL@TBL), we are able to measure the large-scale magnetic field solar twins. Studying the behavior of solar magnetic field, we are able to learn about the mechanisms of magnetic field generation in the dynamo process. Besides convection, various physical parameters affect the dynamo operation, in particular the rotation and mass. We collected signature of the stellar large-scale magnetic field for solar twins and we present here a analysis of seven candidates.
\end{abstract}

Keywords: Stars; spectropolarimetry; star magnetism; twins solar.

\section{Introduction}

The study for solar twins ${ }^{1-2}$ is partially motivated by the search for primary calibrators in stellar astrophysics. Since the Sun cannot be used as a primary calibrator in stellar astrophysics, because it is excessively bright. Observe a calibrating star whose fundamental properties (temperature, luminosity, metallicity, mass, and age) are undistinguishable to solar is critical to the modern astrophysics. Solar twins are an important part to validate stellar interior and evolution models. For example, the observed solar $\mathrm{Li}$ abundance that is 100 times lower relative to that found in meteorites and is not explained by standard stellar evolution models. ${ }^{3}$ Nowadays, only three solar twins are known: 18 Sco from Ref. 4, HD 98618 from Ref. 5, and HIP 100963 from Ref. 6 the other four are quasi solar twins. On this study, we propose an analysis focused on the investigation of magnetic cycles and dynamo evolution for solar twins. Spectropolarimetric observations of solar twins at slight different ages allows to understanding the dependence of magnetic activity on basic stellar parameters such as age, rotation rate and depth of the convection zone, thus providing the observations data necessary for testing dynamo theories. The targets

* Rio Grande do Norte, Brazil 
for this study are solar twins with slight different evolutionary stages. Our two main science objectives are to determine (i) how the magnetic field evolves from thezero age main sequence to the solar age. (ii) the impact of convective depth and rotation rate on magnetic topologies of cool stars. The availability of ESPaDOnS opens an exceptional possibility to study the magnetic properties of Sun-like stars by means of spectropolarimetric observations.

\section{The sample and analysis}

For this study, we selected the solar twins and the quasi solar twins HIP 55459, HIP 41526, HIP 9349, HIP 35185, HIP 43557, HIP 56948 and 18 Sco from a sample of 108 solar analog stars observed with the spectropolarimeter ESPaDOnS at CFHT (Canada-France-Hawaii Telescope) and the Narval at TBL (Telescope Bernard Lyot). The fundamental parameters for our stellar sample are in give on the Table 1. The selected stars are in the mass range of $0.975 \leq M / M_{\odot} \leq 1.025$ with the same solar spectral classification. The effective temperatures obtained for this analysis were derived from Ref. 6. The luminosity was obtained from the parallaxes and apparent visual magnitude $\mathrm{V}$ and bolometric magnitudes computed from HIPPARCOS satellite. The longitudinal component of the magnetic field for our stars was obtained from the Least Squares Deconvolution (LSD) method. ${ }^{7-8}$

\section{Discussion}

On the Fig. 1, we represent the distribution of solar twins on the HR diagram. The different symbol denotes the status of the magnetic field detection. Filled square shows detected magnetic field. Marginal detection represented by inverted triangle and stars without detection by open square. Even, if all twins were practically at the same evolutionary phase as the sun, we see through the Fig. 1 that small changes in internal parameters generate large changes on the longitudinal magnetic field intensity. The spread of magnetic fields values could be also lies to the fact that these stars have different magnetic cycles phase. The Fig. 2 shows the magnetic field intensity as a function of age and we identify a suddenly drop of the magnetic

Table 1. Star parameters to solar twins.

\begin{tabular}{ccccc}
\hline HIP & $T_{\text {eff }}(\mathrm{K})$ & $\log \left(L / L_{\odot}\right)$ & Age $(\mathrm{Gyr})$ & $\left|B_{l}\right|$ \\
Sol & 5780 & 0.00 & 4.5 & 0.5 \\
56948 & 5785 & 0.095 & 4.71 & 0.2 \\
55459 & 5812 & 0.038 & 3.81 & 0.6 \\
41526 & 5801 & 0.026 & 2.64 & 0.9 \\
9349 & 5788 & 0.015 & 2.52 & 7.4 \\
35185 & 5793 & -0.002 & 0.82 & 6.9 \\
43557 & 5805 & 0.056 & 4.18 & 1.7 \\
18 Sco & 5768 & 0.039 & 4.7 & 3.6 \\
\hline
\end{tabular}




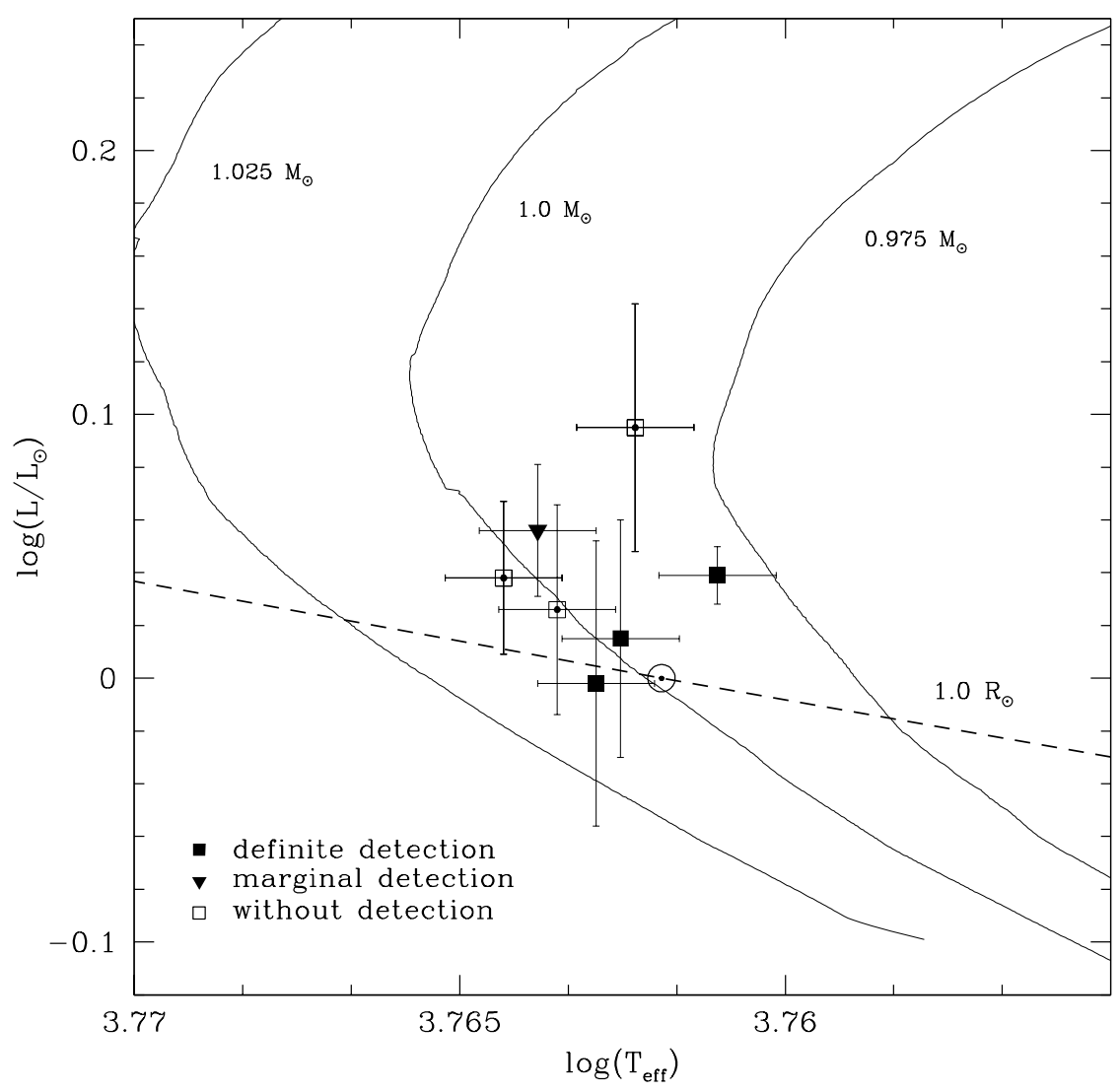

Fig. 1. Distribution of solar twins on the HR diagram. The symbol represents the twins with magnetic detected (square), marginal detection (inverted triangle) and without detection (open square).

field intensity around 2 Gyrs. We are currently examining the fundamental stellar parameters that can explain such a sudden decrease.

\section{Conclusions}

We has studied the magnetic field for a sample of 3 solar twins and 4 quasi solar twins. These stars are known to be the top seven known solar twins. The snapshot magnetic field observations of solar twins from NARAVAL and ESPaDoNS surveys shows a spread in the values of large scale magnetic field. Stellar parameters such as rotation must be the root cause to magnetic field intensity dispersion. 


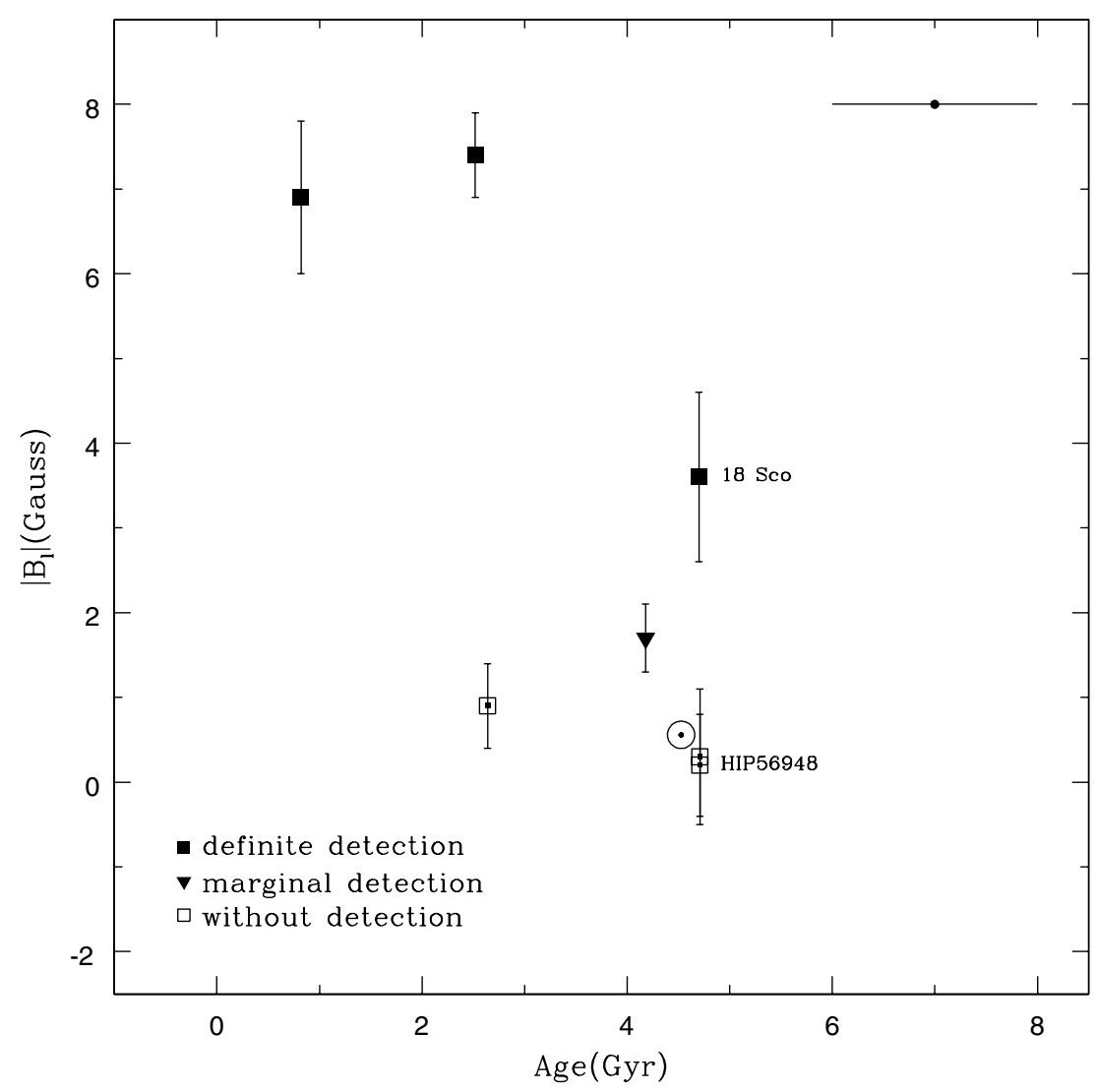

Fig. 2. Magnetic field intensity as a function of Age for our star sample. The symbols follows as in the previous figure. The age were computed as describe from Ref. 9.

\section{References}

1. G. Cayre de Strobel, A\&ARv 7, 243 (1996).

2. G. Cayre de Strobel and C. Bentolila, A\&A 211, 324 (1989).

3. P. V. Foukal, Solar Astrophysics, 2nd rev. edn. (Wiley-VCH, Berlin, 2004), p. 186.

4. G. F. Porto de Mello and L. da Silva, ApJ 482L, 89 (1997).

5. J. Meléndez and I. Ramírez, ApJ 669L, 89 (2007).

6. Y. Takeda, S. Kawanomoto, S. Honda, H. Ando and T. Sakurai, A\&A 468, 663 (2007).

7. P. Petit, B. Dintrans, S. K. Solanki, J.-F. Donati, M. Aurire, F. Lignieres, J. Morin, F. Paletou, J. Ramirez, C. Catala and R. Fares, MNRAS 388, 80 (2008).

8. J.-F. Donati, M. Semel, B. D. Carter, D. E. Rees and A. C. Cameron, MNRAS 291, 658 (1997).

9. J. D. do Nascimento Jr, M. Castro, J. Meléndez, M. Bazot, S. Théado, G. F. Porto de Mello and J. R. de Medeiros, A\&A 501, 687 (2009). 